\title{
GaN Schottky Diode with TiW Electrodes on Silicon Substrate Based on AlN/AlGaN Buffer Layer
}

\author{
Sheng-Po Chang \\ Institute of Microelectronics \& Department of Electrical Engineering, Center for Micro/Nano Science and Technology, \\ Advanced Optoelectronic Technology Center, National Cheng Kung University, Tainan 70101, Taiwan \\ Correspondence should be addressed to Sheng-Po Chang, changsp@mail.ncku.edu.tw
}

Received 30 May 2012; Revised 12 November 2012; Accepted 26 November 2012

Academic Editor: Yong Yang

Copyright ( $(2012$ Sheng-Po Chang. This is an open access article distributed under the Creative Commons Attribution License, which permits unrestricted use, distribution, and reproduction in any medium, provided the original work is properly cited.

We report the fabrication of GaN Schottky photodiodes (PDs) on $\mathrm{Si}(111)$ substrates coated with an AlN/AlGaN buffer multilayer. It was found that their dark current was much smaller than that of identical devices prepared on sapphire substrates. With an incident wavelength of $359 \mathrm{~nm}$, the maximum responsivity of the $\mathrm{n}^{-}$-GaN Schottky photodetectors with TiW contact electrodes was $0.1544 \mathrm{~A} / \mathrm{W}$, corresponding to a quantum efficiency of $53.4 \%$. For a given bandwidth of $1 \mathrm{kHz}$ and bias of $5 \mathrm{~V}$, the resultant noise equivalent power (NEP) of $\mathrm{n}^{-}-\mathrm{GaN}$ Schottky photodetectors with TiW electrodes was $1.033 \times 10^{-12} \mathrm{~W}$, corresponding to a detectivity $\left(D^{*}\right)$ of $1.079 \times 10^{12} \mathrm{~cm}-\mathrm{Hz}^{0.5} \mathrm{~W}^{-1}$.

\section{Introduction}

There are numerous applications demanding the usage of ultraviolet (UV) photodetectors (PDs). Both civil and military disciplines require high-performance UV PDs for solar UV monitoring, source calibration, UV astronomy, flame sensors, detection of missile plumes, space-to-space communications, and many other applications. GaN has a wide direct band gap $\left(E_{g}=3.4 \mathrm{eV}\right)$ and a high saturation velocity $\left(v s=2.7 \times 10^{7} \mathrm{~cm} / \mathrm{s}\right)$ and is therefore widely considered to be one of the most promising materials for realizing UV PDs [1-13]. Moreover, this material is also remarkably tolerant of aggressive environments because of its thermal stability and radiation hardness.

Traditionally, GaN-based epitaxial layers are grown on sapphire or $\mathrm{SiC}$ substrates. Sapphire substrates are electrically insulating and poor thermal conductors. On the other hand, although $\mathrm{SiC}$ substrates are comparably good thermal and electrical conductors, their high cost precludes wide use. Compared with sapphire and $\mathrm{SiC}$ substrates, $\mathrm{GaN}$ epitaxial layers on $\mathrm{Si}$ appear to be a reasonably good substitute, not to mention the fact that the use of Si substrates also has the unique advantage of allowing monolithic integration of GaN-based devices with Si-based microelectronics.

Even though it is rather difficult to grow high-quality $\mathrm{GaN}$ epitaxial layers on $\mathrm{Si}$, GaN-based light-emitting diodes (LEDs) and heterostructure field-effect transistors have nevertheless already been realized on Si substrates [1419]. Our earlier report successfully documented the growth of high-quality InGaN/GaN LED epilayers on a Si(111) substrate [20]. Growth of high-quality InGaN/GaN films on a silicon substrate was possible owing to prior deposition of an AlGaN buffer and two high-temperature (HT) AlN interlayers before attempting GaN growth. This particular growth scheme effectively confined the threading dislocation to the vicinity of the interfacial layer in the $\mathrm{AlGaN} / \mathrm{HT}$-AlN buffer layers. In fact, our transmission electron microscopy (TEM) and scanning electron microscopy (SEM) studies carried out earlier had clearly demonstrated a smooth and crack-free GaN surface with a noticeable reduction in threading dislocation density [20].

In this paper, the deposition of titanium tungsten (TiW) metal contacts on GaN surfaces via RF magnetron sputtering is described. The growth of $\mathrm{n}^{-}-\mathrm{GaN}$ epitaxial layers on $\mathrm{Si}$ substrates and the subsequent fabrication of $\mathrm{GaN}$ 
Schottky photodiodes are discussed thereafter, along with their resultant optical and electrical properties.

\section{Experimental}

Epitaxial samples investigated in our study were all grown on $\operatorname{Si}(111)$ substrates using metalorganic chemical vapor deposition (MOCVD) [21-24]. Trimethylgallium (TMGa), trimethylaluminum (TMAl), and ammonia $\left(\mathrm{NH}_{3}\right)$ were used as source materials for gallium $(\mathrm{Ga})$, aluminum $(\mathrm{Al})$, and nitrogen $(\mathrm{N})$, respectively. To obtain a lightly Si-doped $\mathrm{n}$ type $\mathrm{GaN}\left(\mathrm{n}^{-}-\mathrm{GaN}\right)$ structure, a $25-\mathrm{nm}$-thick AlN buffer layer was deposited on a $\mathrm{Si}(111)$ substrate at $1090^{\circ} \mathrm{C}$. Then, two stacks of buffer multilayers were inserted between the 25-nm-thick AlN buffer layer and the topmost 500-nm-thick $1090^{\circ} \mathrm{C}$-grown $\mathrm{n}^{-}-\mathrm{GaN}$ epitaxial layer. Each stack of buffer multilayers consisted of a 30 -nm-thick $540^{\circ} \mathrm{C}$-grown AlN layer, a 50-nm-thick $1090^{\circ} \mathrm{C}$-grown AlN layer, a 60-nm-thick $1090^{\circ} \mathrm{C}$-grown $\mathrm{Al}_{0.3} \mathrm{Ga}_{0.7} \mathrm{~N}$ layer, a 40 -nm-thick $1090^{\circ} \mathrm{C}$ grown $\mathrm{Al}_{0.2} \mathrm{Ga}_{0.8} \mathrm{~N}$ layer, and a 100 -nm-thick $1090^{\circ} \mathrm{C}$-grown undoped-GaN layer.

TiW $(100 \mathrm{~nm})$ contact layers were then deposited on the samples using an RF magnetron sputter system. Because the $\mathrm{Si}(111)$ substrate was heavily doped, the devices were bonded on TO-Can with Ag rubber for ohmic contact. Then, $\mathrm{n}^{-}$-GaN Schottky diodes were fabricated by standard photolithography and a lift-off process. The active area of photodetectors was $1.26 \times 10^{-3} \mathrm{~cm}^{2}$. An HP-4156 semiconductor parameter analyzer was then used to evaluate the dark current-voltage $(I-V)$ characteristics. Spectral responsivity measurements were also performed using a JOBIN-YVON SPEX system equipped with a 450-W xenon arc lamp and a standard synchronous detection scheme. Furthermore, the noise characteristics of the GaN Schottky diodes were measured within a frequency range of $1 \mathrm{~Hz}-1 \mathrm{kHz}$ using a low-noise current preamplifier and a dynamic signal analyzer.

\section{Results and Discussion}

Figure 1 shows the (002) Bragg reflection double-crystal $\mathrm{X}$-ray diffraction (DCXRD) spectra for a $500 \mathrm{~nm} \mathrm{n}^{-}-\mathrm{GaN}$ epitaxial layer prepared on a Si substrate. The full width at half maximum (FWHM) of the $\mathrm{n}^{-}$-GaN epitaxial layer was measured as $232.11^{\prime \prime}$. Figure 2 shows the $I-V$ characteristics of a Schottky diode with a TiW contact electrode. Under a 5$\mathrm{V}$ applied bias, the measured dark current of photodetectors with TiW electrodes was $7.72 \times 10^{-12} \mathrm{~A}$. It should be noted that a relatively small dark leakage current could still be observed even though these III-nitrides layers were grown on a lattice-mismatched silicon substrate. The small dark current was in fact a clear benefit derived from the insertion of two additional stacks of buffer multilayers into the overall device structure. Evidently, by further improving the crystalline quality of GaN films, this scheme alleviated the impact of GaN-Si lattice mismatch imposed on the epitaxial growth [25]. Furthermore, the much smaller dark current in the vertical PDs was most likely due to the high resistivity

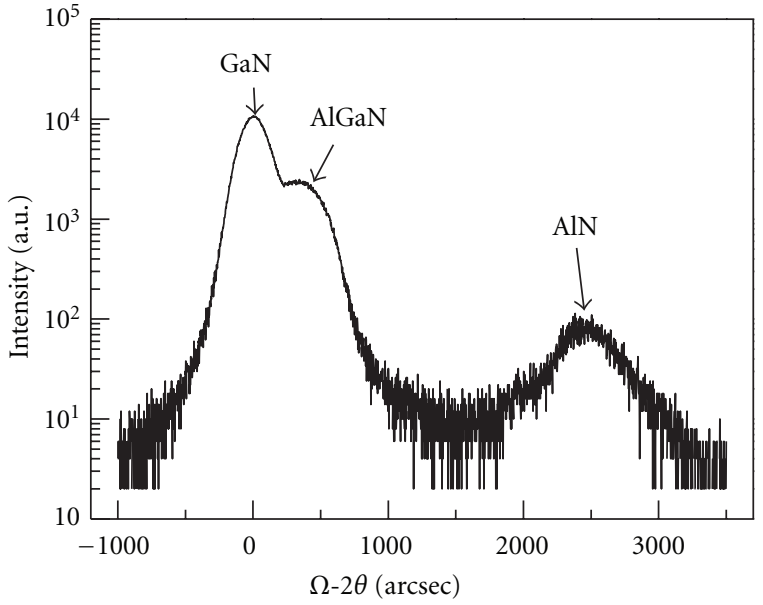

FIgURE 1: The DC-XRD analysis of $\mathrm{n}^{-}-\mathrm{GaN}$ epitaxial structure.

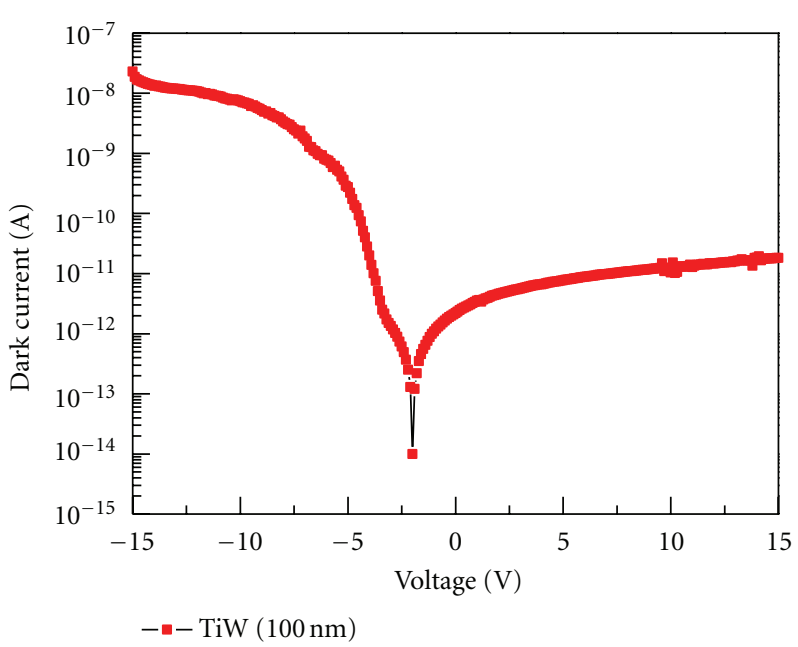

FIgure 2: Dark $I-V$ characteristics of the Schottky diode with TiW contact electrodes.

of the HT-AlN layers used. Consequently, a larger Schottky barrier height between the contact electrode and the epitaxial film was realized.

Figure 3 shows the room temperature spectral responses of PDs under various applied bias voltages. In order to quantify the peak responsivity, the xenon lamp intensity was first measured by a calibrated GaP UV detector. The difference in sensor-detecting areas between the GaP UV detector and our PDs was then determined in order to reliably estimate the PD responsivity.

As shown in Figure 4, the peak responsivity occurred at $359 \mathrm{~nm}$. The maximum responsivity of photodetectors with TiW contact electrodes was $0.1544 \mathrm{~A} / \mathrm{W}$, corresponding to a quantum efficiency of $53.4 \%$. Compared with III-nitride PDs grown on a sapphire substrate $[25,26]$, the smaller peak responsivity observed from samples grown on Si substrates can be directly attributed to a highly defective epitaxial layer. Similar results were also reported by Osinsky et al. [27]. In our case, the responsivity is highly dependent on 


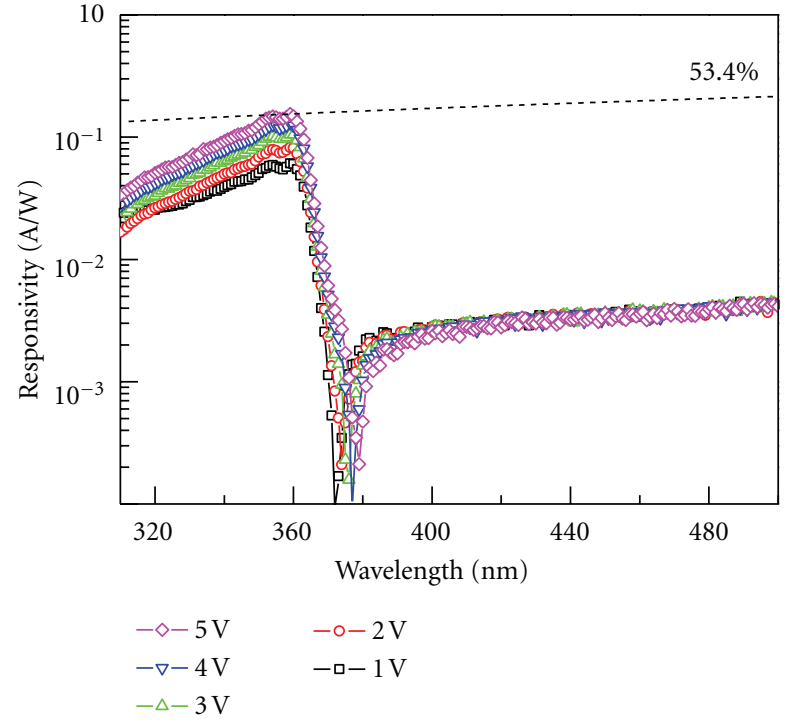

FIGURE 3: Spectral responses of the fabricated detectors with various applied bias.

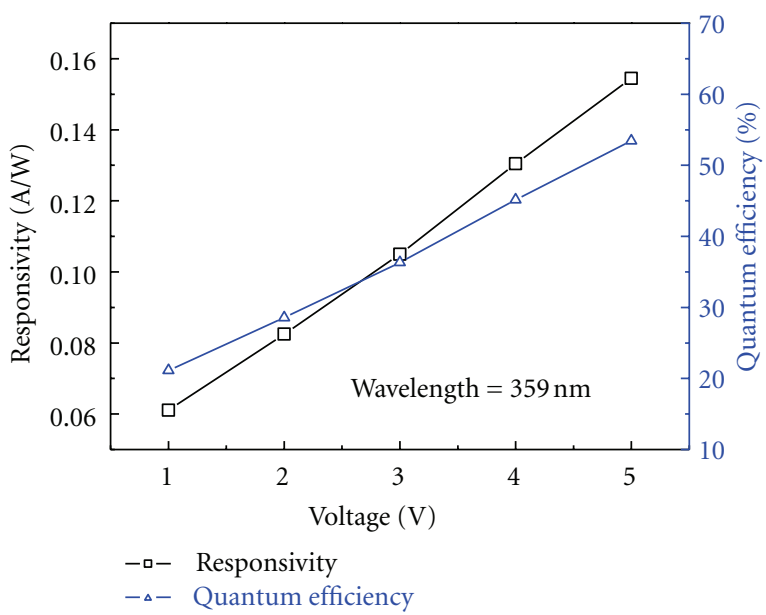

FIGURE 4: Spectral responses and quantum efficiency of photodetectors as functions of applied bias.

the transmittance of the contact electrode, so the higher transmittance of TiW contributes in part to the larger responsivity obtained [25]. In addition, the rejection ratio was determined as the ratio between the spectral responsivity measured at $359 \mathrm{~nm}$ and at $385 \mathrm{~nm}$. With an applied voltage of $5 \mathrm{~V}$, the rejection ratio was 114.4 for photodetectors with TiW contact electrodes. The large rejection ratio can be attributed in part to a high light transparency of TiW electrodes. Figure 4 depicts the peak responsivity $(359 \mathrm{~nm})$ and quantum efficiency of PDs on $\mathrm{Si}$ as a function of bias.

Similar results were also found for AlGaN metalsemiconductor-metal PDs prepared on an Si substrate [28]. The higher voltage enhances the penetration of electronhole pairs through the grain boundary so as to effectively facilitate the collection by electrodes of these charge carriers.

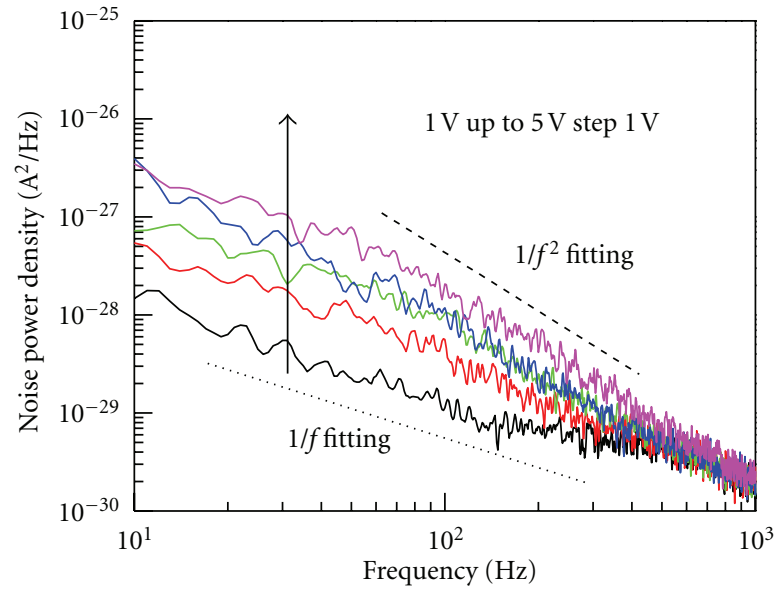

FIGURE 5: Measured noise power densities of the GaN Schottky diode with TiW contact electrodes.

The observation of bias-dependent responsivity of PDs-onSi was also reported by Osinsky et al. [27].

Figure 5 shows the measured noise power density of the GaN Schottky photodetectors with TiW contact electrodes. Noise curves reveal that $1 / f$ (flicker) is a dominant noise mechanism, which is expected for Schottky photodetectors operating at low frequency. Moreover, the noise curves obey the relation of Hooge-type equation with a fitting parameter $\alpha$. It should be noted that the measured low-frequency noise was classified as a $1 / f$-type $(\alpha=1)$ noise for low bias and a $1 / f^{2}$-type $(\alpha=2)$ noise for high bias. For a specified bandwidth $(B)$, the total square of noise current, $\left\langle i_{n}\right\rangle^{2}$, can be determined by integrating the noise power density $S_{n}(f)$ such that

$$
\left\langle i_{n}\right\rangle^{2}=\int S_{n}(f) d f
$$

On the other hand, the noise equivalent power (NEP) can be calculated by

$$
\mathrm{NEP}=\sqrt{\frac{\left\langle i_{n}\right\rangle^{2}}{R}}
$$

where $R$ is the responsivity of the PDs. Furthermore, the normalized detectivity $\left(D^{*}\right)$ can be determined by

$$
D^{*}=\frac{\sqrt{A} \sqrt{B}}{\mathrm{NEP}},
$$

where $A$ is the area of the photodetector and $B$ is the bandwidth. For a given bandwidth of $1 \mathrm{kHz}$ and a given bias of $5 \mathrm{~V}$, the corresponding noise equivalent power of Schottky photodetectors with TiW electrodes was $1.033 \times$ $10^{-12} \mathrm{~W}$. These values in turn led to the calculated detectivity $\left(D^{*}\right)$ of $1.079 \times 10^{12} \mathrm{~cm}-\mathrm{Hz}^{0.5} \mathrm{~W}^{-1}$. A smaller current density was responsible for the smaller NEP observed for Schottky photodetectors on Si. It should be noted that on an average, higher $D^{*}$ was obtained compared with that of devices with similar interdigitated electrode dimensions fabricated on sapphire substrates $[25,29]$. These results were again 
attributed to the smaller noise power density of the PDs prepared on $\mathrm{Si}(111)$ substrates. The benchmark values realized for the noise and detectivity of our devices fabricated on silicon show that these Schottky photodetectors are well suited for low-noise applications.

\section{Summary}

In summary, GaN Schottky photodiodes were prepared on $\mathrm{Si}(111)$ substrates with an AlN/AlGaN buffer multilayer. It was found that the dark current of PDs fabricated on $\mathrm{Si}$ substrates was substantially smaller than that of identical devices prepared on sapphire substrates. With an incident wavelength of $359 \mathrm{~nm}$, the maximum responsivity of the $\mathrm{n}^{-}$GaN Schottky photodetectors with TiW contact electrodes was $0.1544 \mathrm{~A} / \mathrm{W}$, corresponding to a quantum efficiency of $53.4 \%$. For a given bandwidth of $1 \mathrm{kHz}$ and a given bias of $5 \mathrm{~V}$, the corresponding noise equivalent power (NEP) of $\mathrm{n}^{-}$-GaN Schottky photodetectors with TiW electrodes was $1.033 \times 10^{-12} \mathrm{~W}$. Finally, the detectivity $\left(D^{*}\right)$ was determined, therefore, to be $1.079 \times 10^{12} \mathrm{~cm}-\mathrm{Hz}^{0.5} \mathrm{~W}^{-1}$.

\section{Acknowledgments}

The authors would like to thank the National Science Council and Bureau of Energy, Ministry of Economic Affairs of Taiwan, Taiwan, for the financial support under Contracts no. 100-2221-E-006-18 and 101-D0204-6, and the LED Lighting Research Center of NCKU for the assistance of device characterization. This work was also supported in part by the Center for Frontier Materials and Micro/Nano Science and Technology, the National Cheng Kung University, Taiwan, as well as the Advanced Optoelectronic Technology Center, the National Cheng Kung University, under projects from the Ministry of Education.

\section{References}

[1] Y. Z. Chiou, Y. K. Su, S. J. Chang et al., "High detectivity InGaN-GaN multiquantum well p-n junction photodiodes," IEEE Journal of Quantum Electronics, vol. 39, no. 5, pp. 681685, 2003.

[2] E. Monroy, E. Muňoz, F. J. Sánchez et al., "High-performance GaN p-n junction photodetectors for solar ultraviolet applications," Semiconductor Science and Technology, vol. 13, no. 9, p. 1042, 1998.

[3] G. Y. Xu, A. Salvador, W. Kim et al., "High speed, low noise ultraviolet photodetectors based on $\mathrm{GaN}$ p-i-n and $\mathrm{AlGaN}(\mathrm{p})$ GaN(i)-GaN(n)structures," Applied Physics Letters, vol. 71, no. 15 , p. $2154,1997$.

[4] M. Mosca, J. L. Reverchon, N. Grandjean, and J. Y. Duboz, "Multilayer (Al,Ga)N structures for solar-blind detection," IEEE Journal on Selected Topics in Quantum Electronics, vol. 10, no. 4, pp. 752-758, 2004.

[5] N. Biyikli, I. Kimukin, O. Aytur, and E. Ozbay, "Solar-blind A1GaN-based p-i-n photodiodes with low dark current and high detectivity," IEEE Photonics Technology Letters, vol. 16, no. 7, pp. 1718-1720, 2004.

[6] N. Biyikli, I. Kimukin, T. Tut, T. Kartaloglu, O. Aytur, and E. Ozbay, "High-speed characterization of solar-blind
$\mathrm{Al}_{x} \mathrm{Ga}_{1-x} \mathrm{~N}$ p-i-n photodiodes," Semiconductor Science and Technology, vol. 19, no. 11, pp. 1259-1262, 2004.

[7] G. Parish, S. Keller, P. Kozodoy et al., "High-performance $(\mathrm{Al}, \mathrm{Ga}) \mathrm{N}$-based solar-blind ultraviolet $\mathrm{p}-\mathrm{i}-\mathrm{n}$ detectors on laterally epitaxially overgrown GaN," Applied Physics Letters, vol. 75, no. 2, pp. 247-249, 1999.

[8] A. Osinsky, S. Gangopadhyay, R. Gaska et al., "Low noise p- $\pi$ n GaN ultraviolet photodetectors," Applied Physics Letters, vol. 71, no. 16, pp. 2334-2336, 1997.

[9] V. Adivarahan, G. Simin, J. W. Yang et al., " $\mathrm{SiO}_{2}$ lateralgeometry GaN transparent Schottky-barrier detectors," Applied Physics Letters, vol. 77, no. 6, pp. 863-865, 2000.

[10] S. J. Chang, M. L. Lee, J. K. Sheu et al., "GaN metalsemiconductor-metal photodetectors with low-temperatureGaN cap layers and ITO metal contacts," IEEE Electron Device Letters, vol. 24, no. 4, pp. 212-214, 2003.

[11] O. Katz, V. Garber, B. Meyler, G. Bahir, and J. Salzman, "Gain mechanism in GaN Schottky ultraviolet detectors," Applied Physics Letters, vol. 79, no. 10, pp. 1417-1419, 2001.

[12] E. Monroy, T. Palacios, O. Hainaut, F. Omnès, F. Calle, and J. F. Hochedez, "Assessment of GaN metal-semiconductormetal photodiodes for high-energy ultraviolet photodetection," Applied Physics Letters, vol. 80, no. 17, p. 3198, 2002.

[13] J. L. Pau, C. Rivera, E. Muňoz et al., "Response of ultralow dislocation density GaN photodetectors in the near- and vacuum-ultraviolet," Journal of Applied Physics, vol. 95, no. 12, p. $8275,2004$.

[14] C. F. Shih, N. C. Chen, C. A. Chang, and K. S. Liu, "Blue, Green and White InGaN Light-Emitting Diodes Grown on Si," Japanese Journal of Applied Physics, vol. 44, pp. L140-L143, 2005.

[15] H. Ishikawa, K. Asano, B. Zhang, T. Egawa, and T. Jimbo, "Improved characteristics of GaN-based light-emitting diodes by distributed Bragg reflector grown on Si," Physica Status Solidi, vol. 201, no. 12, pp. 2653-2657, 2004.

[16] S. Iwakami, M. Yanagihara, O. Machida et al., "AlGaN/GaN heterostructure field-effect transistors (HFETs) on Si substrates for large-current operation," Japanese Journal of Applied Physics, vol. 43, pp. L831-L833, 2004.

[17] A. Curutchet, N. Malbert, N. Labat et al., "Low frequency drain noise comparison of AlGaN/GaN HEMT's grown on silicon, SiC and sapphire substrates," Microelectronics Reliability, vol. 43, no. 9-11, pp. 1713-1718, 2003.

[18] P. Javorka, A. Alam, M. Marso et al., "Material and device issues of AlGaN/GaN HEMTs on silicon substrates," Microelectronics Journal, vol. 34, no. 5-8, pp. 435-437, 2003.

[19] V. Hoël, Y. Guhel, B. Boudart et al., "Static measurements of GaN MESFETs on (111) Si substrates," Electronics Letters, vol. 37, no. 17, pp. 1095-1096, 2001.

[20] Y. P. Hsu, S. J. Chang, W. S. Chen, J. K. Sheu, J. Y. Chu, and C. T. Kuo, "Crack-free high-brightness InGaN/GaN LEDs on Si(111) with initial AlGaN buffer and two LT-Al interlayers," Journal of the Electrochemical Society, vol. 154, no. 3, pp. H191H193, 2007.

[21] S. J. Chang, W. C. Lai, Y. K. Su, J. F. Chen, C. H. Liu, and U. H. Liaw, "InGaN-GaN multiquantum-well blue and green lightemitting diodes," IEEE Journal of Selected Topics in Quantum Electronics, vol. 8, no. 2, pp. 278-283, 2002.

[22] S. J. Chang, C. S. Chang, Y. K. Su et al., "Highly reliable nitridebased LEDs with SPS+ITO upper contacts," IEEE Journal of Quantum Electronics, vol. 39, no. 11, pp. 1439-1443, 2003.

[23] S. J. Chang, C. H. Kuo, Y. K. Su et al., "400-nm InGaN-GaN and InGaN-AlGaN multiquantum well light-emitting diodes," 
IEEE Journal on Selected Topics in Quantum Electronics, vol. 8, no. 4, pp. 744-748, 2002.

[24] S. J. Chang, C. S. Chang, Y. K. Su et al., "Nitride-based flip-chip ITO LEDs," IEEE Transactions on Advanced Packaging, vol. 28, no. 2, pp. 273-277, 2005.

[25] C. K. Wang, S. J. Chang, Y. K. Su et al., "GaN MSM UV photodetectors with titanium tungsten transparent electrodes," IEEE Trans. Electron Dev, vol. 53, no. 1, pp. 38-42, 2006.

[26] Y. Z. Chiou, "Nitride-based p-i-n bandpass photodetectors," IEEE Electron Device Letters, vol. 26, no. 3, pp. 172-174, 2005.

[27] A. Osinsky, S. Gangopadhyay, J. W. Yang, and R. Gaska, "Visible-blind GaN Schottky barrier detectors grown on Si(111)," Applied Physics Letters, vol. 72, no. 5, p. 551, 1998.

[28] Y. Z. Chiou, Y. C. Lin, and C. K. Wang, "A1GaN photodetectors prepared on Si substrates," IEEE Electron Device Letters, vol. 28, no. 4, pp. 264-266, 2007.

[29] Y. K. Su, P. C. Chang, C. H. Chen et al., "Nitride-based MSM UV photodetectors with photo-chemical annealing Schottky contacts," Solid-State Elctron, vol. 49, no. 3, pp. 459-463, 2005. 

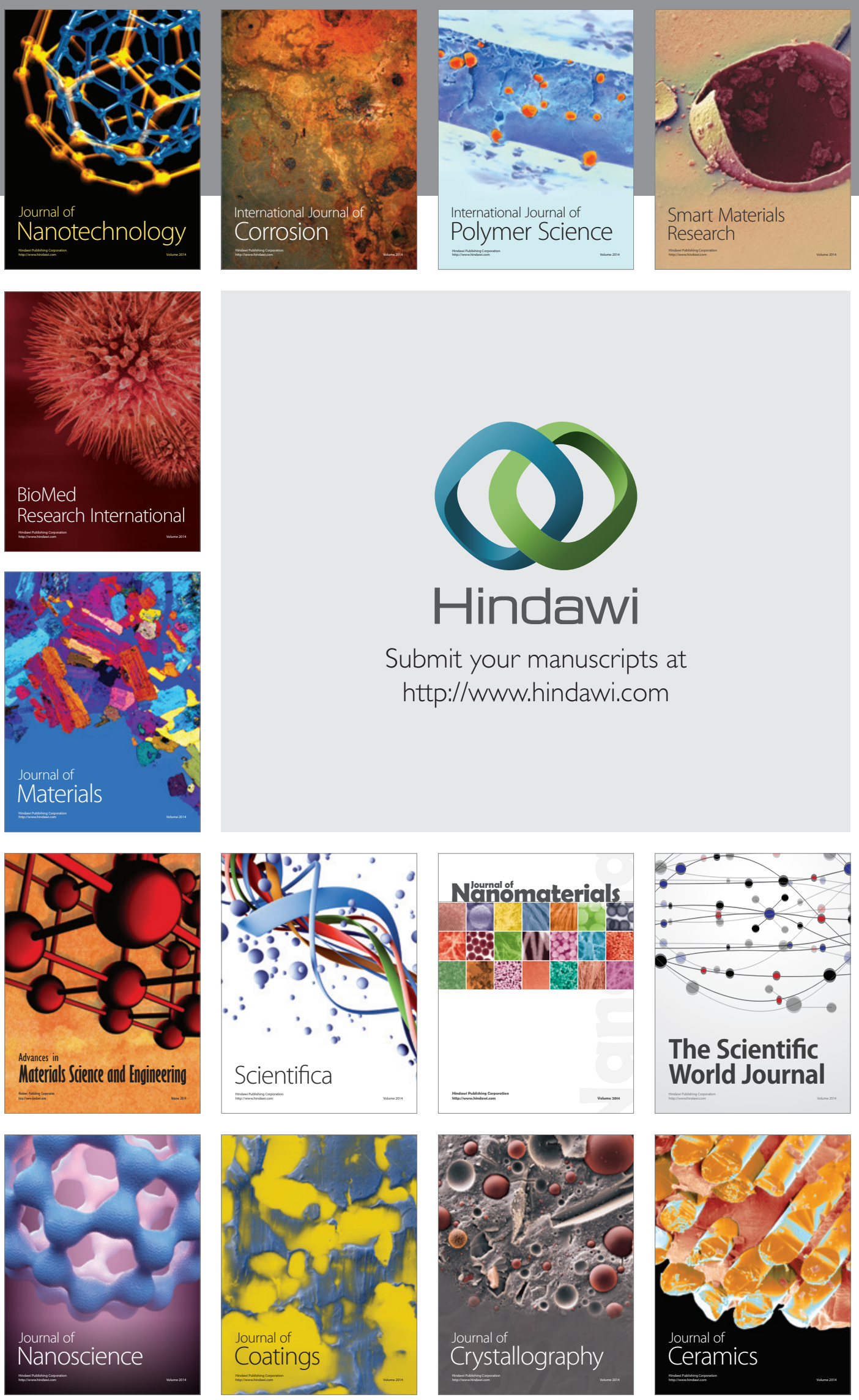

The Scientific World Journal

Submit your manuscripts at

http://www.hindawi.com

\section{World Journal}

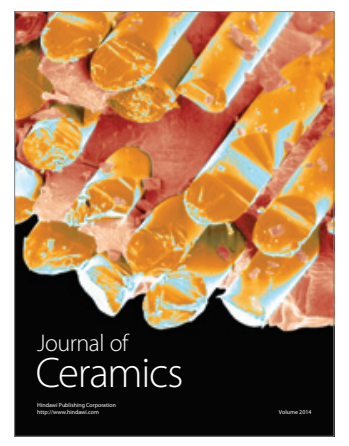

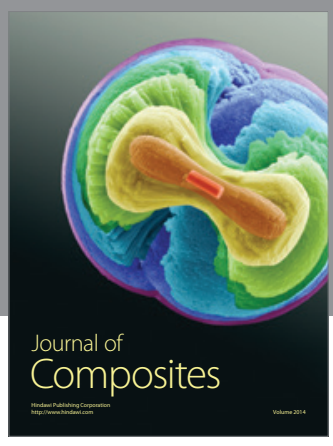
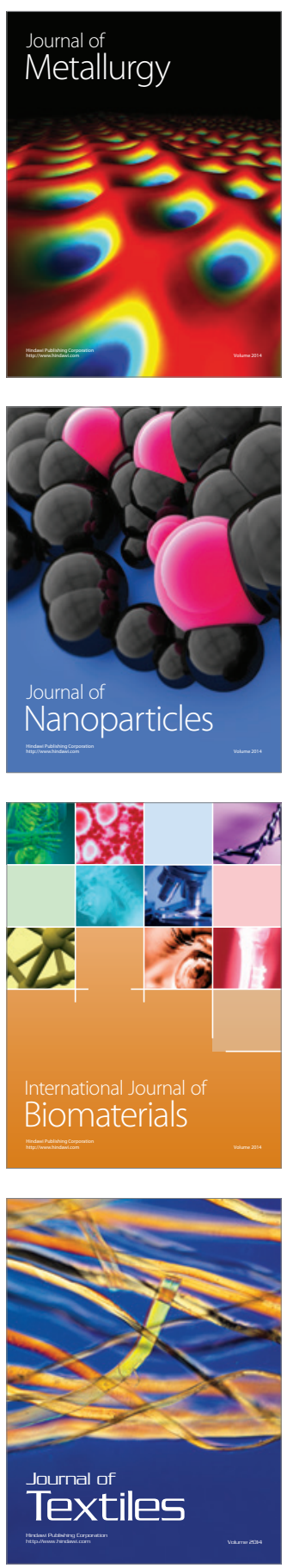